SLAC-PUB-8242

September 1999

\title{
Non-linear Mode Coupling and Saw-Tooth Instability
}

\author{
S. Heifets
}

Contributed to Workshop on Instabilities in High Intensity Hadron Beams in Rings, 6/28/99—7/1/99, Brookhaven, NV, USA

Stanford Linear Accelerator Center, Stanford University, Stanford, CA 94309

Work supported by Department of Energy contract DE-AC03-76SF00515. 
SLAC-PUB-8242

September 1999

\title{
Non-linear Mode Coupling and Saw-Tooth Instability
}

\author{
S. Heifets \\ Stanford Linear Accelerator Center, Stanford University, P.O. Box 4349, Stanford, CA 94309
}

\begin{abstract}
Dynamics of the longitudinal relaxation oscillations of a single bunch above the threshold of microwave instability is discussed.
\end{abstract}

\section{INTRODUCTION}

Usually, beam dynamics in accelerators is linear by design and nonlinear effects are considered in context of long-run single particle stability. There are, however, a number of phenomena where nonlinear behavior is essential:

1. Beam-beam blow-up and O-type oscillations,

2. Beam-Ion instability

3. Saturation of single/multi-bunch instabilities,

4. Saw-tooth instability,

5. Relaxation oscillations in the beam interacting with high-Q resonator (SPEAR, J. Sebek, C. Limborg)

6. Transverse relaxation oscillations (K. Harkay, Argon).

Similar problems exist and were studied in hadron machines (P.Colestock).

The 1D saw-tooth instability [1] provide a simple case for study of these phenomena. It was observed in many laboratories as periodic relaxation oscillations of the rms bunch length with corresponding excitation of the second- o third harmonics of synchrotron frequency in the bunch spectra. The instability manifests itself in seemingly simple situation of a single bunch under steady-state external conditions and can be considered as onset of the microwave instability. Hopefully, approach described below may be relevant to hadron machines.

\section{HAISSINSKI SOLUTION}

At low current, a bunch can be considered as $N_{b}$ uncorrelated individual particles oscillating in an one-dimensional time-independent potential of the rf bucket with 
the synchrotron frequency $\omega_{0 s} / 2 \pi$. The particle motion is described in terms of dimensionless canonical variables

$$
x=z / \sigma_{0}, \quad p=-\delta / \delta_{0}, \quad\{x, p\}=1
$$

where $z$ is position of a particle in respect with bunch centroid $(z>0$ in the head of the bunch), $\delta=\Delta E / E, \sigma_{0}$ and $\delta_{0}$ are the rms bunch length and the rms energy spread in a bunch at zero current. It is convenient to use also dimensionless time $s=\omega_{0 s} t$.

At large number of particles per bunch $N_{b}$, the nonlinearity of the potential is dominated by interaction of particles through a longitudinal wake-field excited by the beam. The wake field is characterized by the longitudinal beam impedance $Z(\omega)$. The Hamiltonian $H(x, p, s)=\frac{p^{2}}{2}+V(x, s)$ where the self-consistent potential depends on the distribution function $\rho, \int \rho d x d p=1$,

$$
V(x, s)=\frac{x^{2}}{2}+\lambda \int_{x}^{\infty} d x^{\prime} d p^{\prime} \rho\left(x^{\prime}, p^{\prime}, s\right) S\left(x^{\prime}-x\right)
$$

and

$$
S(x)=\frac{4 \pi}{Z_{0}} \int \frac{d \omega}{2 \pi i} \frac{Z(\omega)}{\omega}\left[1-e^{-i\left(\omega \sigma_{0} / c_{0}\right) x}\right] .
$$

Here $Z_{0}=4 \pi / c_{0}=120 \pi$ Ohm is impedance of vacuum,

$$
\lambda=\frac{N_{b} r_{0}}{2 \pi R \gamma \alpha \delta_{0}^{2}}
$$

$r_{0}$ is electron classical radius, $2 \pi R$ is machine circumference, and $\alpha$ is momentum compaction.

In a simple case of a narrow-band impedance centered at $\omega_{r}$ with shunt impedance $R_{r}$ and quality factor $Q_{r}$,

$$
S(x)=\frac{4 \pi R_{r}}{Z_{0} Q_{r} \zeta} \sin [\mu \zeta x] e^{-\mu x / 2 Q_{r}},
$$

where $\mu=\omega_{r} \sigma_{l} / c$, and $\zeta=\sqrt{1-1 /\left(2 Q_{r}\right)^{2}}$. This impedance is used below.

Diffusion and radiation damping caused by synchrotron radiation are described by the Fokker-Plank equation for the distribution function $\rho$. In steady-state, there is Haiisinski solution [2] $\rho_{H}(x, p)=\left(1 / Z_{H}\right) e^{-H_{H}(x, p)}$ where $H_{H}$ depends on $\rho_{H}$ in a self-consistent way, and temperature $T=1$ in the chosen variables.Explicit expression for Haiisinski distribution and for self-consistent potential $U_{H}(x)$ can be obtained numerically.

In this note we consider relatively small $\lambda$ where $U_{H}(x)$ has only one minimum and coherent frequency shift is small. In this case, neither the well-known mode coupling instability nor the Baartman-Dyachkov mechanism [3] can explain the instability. 
Because phase mixing is fast process, it is convenient to use action-angle variables $J, \phi$ introduced in such a way that, in the steady-state $\rho_{H}$, Hamiltonian $H_{H}(p, x)=H_{H}(J)$. Then $\rho_{H}$ is independent on $\phi$ and normalized by $d J d \phi \rho_{H}(J)=$ 1. Numerical integration $J=(1 / \pi) \int d x \sqrt{2\left(H-U_{H}(x)\right)}$ where integral is taken between turning points, gives $J(H)$ and defines frequency $\omega_{H}(J)=d H_{H} / d J$.

In the time-dependent case,

$$
\rho(J, \phi, s)=\rho_{H}(J)+\sum_{n} \rho_{n}(J) e^{i n \phi}, \quad H=H_{H}(J)+\sum_{n} U_{n}(J, s) e^{i n \phi},
$$

where

$$
U_{n}(J, s)=\lambda \int d J^{\prime} d \phi^{\prime} \rho_{m}(J, s) R_{m, m^{\prime}}\left(J, J^{\prime}\right) .
$$

Note that $U_{n}$ is defined only by amplitudes $\rho_{m}$ while, by definition of $J, \phi$ variables, all azimuthal harmonics $U_{n}$ generated by $\rho_{H}$ are canceled by terms arising from $p^{2} / 2+x^{2} / 2$ part of the Hamiltonian.

Coefficients $R_{m, m^{\prime}}$ are related to the impedance

$$
\begin{gathered}
R_{m, m^{\prime}}\left(J, J^{\prime}\right)=-\frac{4 \pi}{Z_{0}} \int \frac{d \omega}{2 \pi i} \frac{Z(\omega)}{\omega} C_{m}(J, \omega) C_{m^{\prime}}^{*}\left(J^{\prime}, \omega\right), \\
C_{m}(J, \omega)=\int \frac{d \phi}{2 \pi} e^{-i m \phi} e^{i x(J, \phi)} .
\end{gathered}
$$

where $x(J, \phi)$ is particle trajectory in the self-consistent Haiisinski potential. From the symmetry $t \rightarrow-t$ it follows that $x(J,-\phi)=x(J, \phi)$. Then $R_{m, m^{\prime}}$ are real what follows from the symmetry of impedance $Z^{*}(-\omega)=Z\left(\omega^{*}\right)$.

\section{FOKKER-PLANK EQUATION. LINEAR APPROXIMATION}

The Fokker-Plank equation in $J, \phi$ variables gives for harmonics $\rho_{n}(J, s), n=$ $0,1, .$.

$$
\frac{\partial \rho_{n}(J, s)}{\partial s}+i n \omega_{H}(J) \rho_{n}-i n \rho_{H}^{\prime}(J) U_{n}+i \sum\left[\frac{\partial U_{m}}{\partial J}(n-m) \rho_{n-m}-\frac{\partial \rho_{n-m}}{\partial J} m U_{m}\right]=\gamma_{0} F_{n} .
$$

Here $\rho_{H}^{\prime}=\frac{\partial \rho_{H}(J)}{\partial J}, \gamma_{0}$ is synchrotron radiation damping, and $F_{n}$ describes diffusion and damping. Neglecting anharmonic terms of trajectory and terms of the order of $\gamma_{0}$, one gets

$$
F_{n}=\frac{\partial}{\partial J}\left[\frac{J}{\omega_{H}} \frac{\partial \rho_{n}}{\partial J}+J \rho_{n}\right]-\frac{n^{2}}{4 J \omega_{H}} \rho_{n}
$$


For small $\lambda$ (low current), harmonics oscillate with time as $\rho_{n} \propto e^{-i \omega_{H} s}$. Hence, $U_{n}$ is given by the sum of terms oscillating proportional to $R_{m, k} e^{i(m-k) \omega_{H} s}$. Averaging of fast oscillating terms leaves only diagonal term

$$
U_{m}(J)=\lambda \int d J^{\prime} d \phi^{\prime} R_{m m}\left(J, J^{\prime}\right) \rho_{m}\left(J^{\prime}, s\right)
$$

In the linear approximation, all azimuthal modes are independent and each is described by the superposition of radial modes $X_{\nu}$,

$$
\rho_{n}(J, s)=\rho_{H}^{\prime}(J) \sum_{\nu} b_{\nu} X_{\nu}(J) e^{-i\left(\omega_{H}-\bar{\omega}\right) s}
$$

$X_{\nu}$ are eigen vectors of the matrix

$$
\begin{gathered}
M_{m}\left(J, J^{\prime}\right)=2 \pi \lambda R_{m m}\left(J, J^{\prime}\right) \rho_{H}^{\prime}\left(J^{\prime}\right)-\delta\left(J-J^{\prime}\right)\left(\omega_{H}(J)-\bar{\omega}\right), \\
\int d J^{\prime} M_{m}\left(J, J^{\prime}\right) X_{\nu}\left(J^{\prime}\right)=-\nu X_{\nu}(J) .
\end{gathered}
$$

The beam is linearly unstable if at least one of the eigen-values $\nu$ has positive imaginary part $\Gamma_{\nu}=\operatorname{Im}[\nu]>\gamma_{n}$. In Eq. (14) we introduce constant $\bar{\omega}$ so that $\Omega=R e \nu$ is the coherent frequency shift. The diagonal approximation for $R_{m k}$ is valid if $\operatorname{Re\nu }<<\omega_{H} \simeq 1$.

Note, $U_{m}(J, s)=\sum_{\nu}\left(\omega_{H}-\bar{\omega}-\nu\right) b_{\nu} X_{\nu}(J)$.

If $\rho_{H}^{\prime}(J)$ is monotonic function, the kernel of Eq.(15) can be written in a more symmetric form if vectors $\sqrt{\rho_{H}^{\prime}} \tilde{X}_{\nu}(J)$ are used instead of $X_{n u}(J)$. In this form it is easy to see that, apart of Landau damping, the eigen values are real if $R_{m m}$ is symmetric, $R_{m m}\left(J, J^{\prime}\right)=R_{m m}\left(J^{\prime}, J\right)$. Hence, the beam stability depends on the anharmonicity of the trajectories and given by the asymmetric part of $R_{m m}$ [4].

Eq. (15)

$$
\left[\omega_{H}(J)-\bar{\omega}-\nu\right] X_{\nu}(J)=2 \pi \lambda \int d J^{\prime} R_{m m}\left(J, J^{\prime}\right) X_{\nu}\left(J^{\prime}\right)
$$

shows that the structure of the mode is

$$
X_{\nu}(J)=\frac{r_{m}(J)}{\omega_{H}(J)-\bar{\omega}-\nu}
$$

where $r_{m}(J)$ is a smooth function of $J$. Hence, the mode is localized around the resonance value $J_{r}, \omega\left(J_{r}\right)=\bar{\omega}+\Omega$ with the width $\Gamma_{m}$. 


\section{COMPARISON WITH A NONLINEAR OSCILLATOR}

Well above the threshold, large number of unstable interacting modes leads to the turbulent regime of instability.

We are interested in the case where coherent tune shift is by an order of magnitude smaller than the dimensionless synchrotron frequency (which is of the order of one in our variables). In this case, it could be that only single mode is unstable, and interaction of this coherent mode with particles is important. These interaction can be described in the quasi-linear approximation [5].

The hint on how such a small perturbation can lead to non-perturbative effects can be obtained from the behavior of nonlinear oscillator in periodic external field $F=-\epsilon \cos \left(\Omega s+\psi_{0}\right)$. This case is quite analogous to interaction of a particle with an unstable dipole mode of a bunch which produces the periodic perturbation $U_{1} \propto e^{-i \omega_{H} t}$. Effect of this perturbation on particles can be described by the Hamiltonian

$$
H(J, \phi, s)=H_{0}(J)+\frac{\epsilon}{2} \sqrt{\frac{2 J}{\omega_{0}}} \cos \left(\phi-\Omega s-\psi_{0}\right),
$$

where $H_{0}(J)=\omega_{0} J+\kappa J^{2} / 2, \kappa$ is parameter of nonlinearity $\omega(J)=\omega_{0}+\kappa J$. For $\Omega=\omega_{0}+\Delta, \Delta<<\omega_{0}$. The resonance Hamiltonian in the new canonical variables $J, \alpha, \alpha=\phi-\Omega s-\psi_{0}$, is time-independent

$$
H(J, \alpha)=-\Delta J+\frac{\kappa J^{2}}{2}+\frac{\epsilon}{2} \sqrt{\frac{2 J}{\omega_{0}}} \cos \alpha .
$$

There are fixed points (FP) at $\alpha=0$ or $\alpha=\pi$ given by equation

$$
-\Delta+\kappa J \pm \frac{\eta}{\sqrt{J}}=0
$$

where $\eta=\epsilon /\left(2 \sqrt{2 J \omega_{0}}\right)$.

If $\Delta / \kappa<0$, there is one FP at $\alpha=\pi$ for any $\eta$. If $\Delta / \kappa>0$, one FP is located at $\alpha=\pi, J_{r} \simeq \eta^{2 / 3}$ for $\eta>>(\Delta / \kappa)^{3 / 2}$, and $J_{r} \simeq(\Delta / \kappa)$ for $\left.\eta<<(\Delta / \kappa)^{3 / 2}\right)$. For small $\eta<\eta_{\text {max }}=2(\Delta / 3 \kappa)^{3 / 2}$ there are two additional FPs at $\alpha=0$, one of them is stable at $J_{r} \simeq(\kappa / \Delta)^{2} \eta^{2}$, and one is unstable at $J_{r} \simeq \Delta / \kappa$. The stable FP is at the

center of a narrow separatrix with the width $(\delta J)=8 \eta \sqrt{\Delta / \kappa}$, while trajectories of particles outside of the separatrix are circles centered at $J_{r}=(\kappa / \Delta)^{2} \eta^{2}$ and $\alpha=\pi$. The width of the separatrix grows with the amplitude of perturbation $\epsilon$ and more and more particles are trapped inside of the separatrix. Trajectories of particles outside of the separatrix retain in the shrinking area which center shifts to large $J$ at $\alpha=\pi$. This area disappears eventually when amplitude $\eta$ exceeds $\eta_{\max }$.

It is worth noting that the resonance exists at arbitrary small amplitudes $\epsilon$ and the width of the resonance depends on the ratio of $\epsilon$ to nonlinearity $\kappa$ and, hence, can be large even for small amplitudes for small nonlinearities. 
The split of a beam into two beamlets corresponding trapping in the separatrix was observed recently in experiment [6].

Let us consider the same case of external force with Fokker-Plank equation. Harmonics $\rho_{n}$ satisfy Eq. (13) while $U_{n}$ has in this case only one harmonics $U_{1}=$ $U_{-1}^{*}=\eta \sqrt{J} e^{-i \Omega s}$, where $\eta$ may grow $\eta=\eta_{0} e^{\Gamma s}$. Such an analysis, although not self-consistent, may clarify possible effects of the mode coupling.

Equation for $\rho_{1}$ in the linear approximation has a familiar solution $\rho_{1}(J)=$ $\rho_{\text {res }}(J)$,

$$
\rho_{\text {res }}=\frac{\eta \rho_{H}^{\prime}(J) \sqrt{J}}{\left(J-J_{r}-i w\right)} e^{-i \Omega s}
$$

were $J_{r}=\Delta / \kappa$ and the width of the resonance $w=\Gamma / \kappa$. Hence, the linearized equation describes the resonance mode but not modification of the rest of the phase plane.

Let us include now coupling to harmonics $\rho_{0}$ and $\rho_{ \pm 2}$

$$
\dot{\rho}_{1}+i \omega_{H} \rho_{1}-i \rho_{H}^{\prime} U_{1}+2 i \frac{\partial U_{1}^{*}}{\partial J} \rho_{2}-i \frac{\partial \rho_{0}}{\partial J} U_{1}+i \frac{\partial \rho_{2}}{\partial J} U_{1}^{*}=0
$$

Equation

$$
\dot{\rho}_{0}+\gamma_{0} \rho_{0}=i \frac{\partial}{\partial J}\left[U_{1} \rho_{1}^{*}-c . c\right]
$$

can be solved explicitly giving

$$
\rho_{0}=i \frac{\partial}{\partial J}\left[\frac{U_{1} \rho_{1}^{*}}{2 \Gamma}-c . c\right] .
$$

Note, that the condition $\int d J \rho_{0}(J)=0$ gives the boundary condition $U_{1} \rho_{1}=0$ at $J=0$.

Similarly, for $\rho_{2}=\bar{\rho}_{2} e^{-2 i \omega_{H} s+2 \Gamma s}$ we get

$$
\bar{\rho}_{2}=\frac{\bar{U}_{1}^{2}}{\omega_{H}-\Omega-i \Gamma} \frac{\partial}{\partial J}\left[\frac{\bar{\rho}_{1}}{\bar{U}_{1}}\right]
$$

where $\bar{\rho}_{1}=\eta \sqrt{J} f$ is defined as $\rho_{1}=\bar{\rho}_{1} e^{-i \omega_{H} s+\Gamma s}$.

Function $f(J)$, satisfies equation

$$
\left(J-J_{r}-i w\right) f=\rho_{H}^{\prime}(J)+\frac{i \eta^{2}}{2 w} \frac{\partial^{2}}{\partial J^{2}}\left[J f^{*}-c . c .\right]-\left\{\frac{\eta^{2}(\partial f / \partial J)}{J-J_{r}-i w}+\frac{\partial}{\partial J}\left[\frac{\eta^{2} J(\partial f / \partial J)}{J-J_{r}-i w}\right]\right\} .
$$

Here $J_{r}=\Delta / \kappa$, and $w=\Gamma / \kappa$. The first term in the RHS gives resonance solution of the linearized equation, the second term gives correction due to coupling to $\rho_{0}$, and the last term in curly brackets describes effect of the quadrupole mode. 
For small $\eta$, equation can be solved by iterations substituting the resonance solution in the last two terms. Fig. 1 shows distribution function $\rho_{H}+\rho_{0}$ (left column) and $\rho_{0}$ (right column) vs $J$ perturbed by the dipole (upper row) and quadrupole modes (second row). Parameters $\eta=0.03, w=0.2, J_{r}=0.75, \kappa=0.1$ and $\omega_{H}=0.7+\kappa J$ were used in calculations.
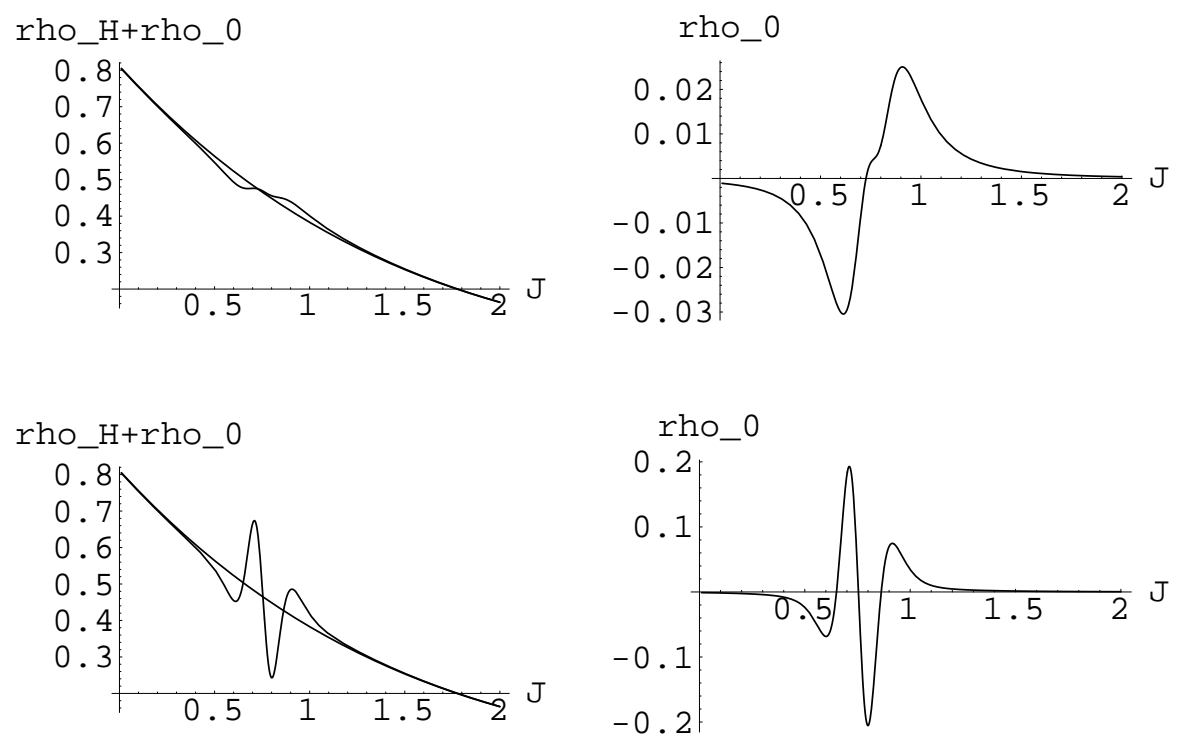

FIGURE 1. Distortion of the distribution function by the coherent modes: top row: by dipole mode, bottom row: quadrupole mode.

The second and the third terms in Eq. (26) are small compared to the first term if $\left(J-J_{r}\right)^{2}<\eta \sqrt{J_{r}}$ what corresponds to the width of the separatrix of the nonlinear resonance discussed above.

The dipole mode affects particle distribution flattening it at the resonance, result which is well known in the quasi-linear approximation. Particles with low energy move to the right. As a result, energy of the system increases what leads, in a selfconsistent Vlasov equation, to saturation of the unstable mode. It is interesting, that effect of the quadrupole mode is opposite. This suggest that interaction of two modes may lead to oscillatory regime.

To summarize, we note that linear approximation describes the resonance while the zero-harmonic $\rho_{0}$ may be required to describe the distortion of distribution function due to perturbation of the non-resonance particles by the coherent mode.

\section{NONLINEAR REGIME}

Below certain threshold current, eigen-values of all azimuthal modes in linear approximation are real, the beam is stable, and is described by Haissinski distribution. Just above the threshold, there is a single unstable mode. Distribution 
function still is a series of eigen-functions Eq. (13) but with time-dependent coefficients $b_{n}(s)$. The non-trivial phenomena above the threshold corresponds to the non-linear interaction of the modes. Which one of the azimuthal modes become unstable first depends on the character of the impedance and the answer can be obtained from the linear analysis. Usually, it is a competition between dipole $(m=1)$ and quadrupole $(m=2)$ modes while sextupole and higher order modes come into play at higher currents. Considering instability close to the threshold, we can take into account only harmonics $\rho_{m}$ with $m=1,2$ expanding them in eigen vectors $V_{\nu}=\left(X_{\nu}, Y_{\nu}\right.$ of the linear problem

$$
\begin{aligned}
& \rho_{1}(J, s)=\rho_{H}^{\prime}(J) \sum_{\nu} b_{\nu} X_{\nu}(J) e^{-i\left(\omega_{H}-\bar{\omega}\right) s} \\
& \rho_{2}(J, s)=\rho_{H}^{\prime}(J) \sum_{\mu} a_{\mu} Y_{\mu}(J) e^{-2 i\left(\omega_{H}-\bar{\omega}\right) s}
\end{aligned}
$$

where

$$
M_{m}\left(J, J^{\prime}\right)=2 \pi \lambda R_{m m}\left(J, J^{\prime}\right) \rho_{H}^{\prime}\left(J^{\prime}\right)-\delta\left(J-J^{\prime}\right)\left(\omega_{H}(J)-\bar{\omega}\right),
$$

with $m=1,2$, and

$$
\begin{aligned}
& \int d J^{\prime} M_{1}\left(J, J^{\prime}\right) X_{\nu}\left(J^{\prime}\right)=-\nu X_{\nu}(J), \\
& \int d J^{\prime} M_{2}\left(J, J^{\prime}\right) Y_{\mu}\left(J^{\prime}\right)=-\mu Y_{\mu}(J) .
\end{aligned}
$$

The radial amplitudes satisfy equations

$$
\begin{aligned}
& \dot{b}_{\nu}+\left(i \nu+\gamma_{d}\right) b_{\nu}+i \sum_{\mu, \sigma} C_{\mu, \sigma}^{\nu} a_{\mu} b_{\sigma}^{*}+i \sum_{\sigma} d_{\nu, \sigma} b_{\sigma}=0 \\
& \dot{a}_{\mu}+\left(2 i \mu+\gamma_{q}\right) a_{\mu}+i \sum_{\sigma, \lambda} g_{\sigma, \lambda}^{\mu} b_{\lambda} b_{\sigma}+i \sum_{\sigma} f_{\mu, \sigma} a_{\sigma}=0
\end{aligned}
$$

Here we used orthogonality $\int d J \bar{X}_{\nu} X_{\nu^{\prime}}=\delta_{\nu, \nu^{\prime}}$, and $\int d J \bar{Y}_{\mu} Y_{\mu^{\prime}}=\delta_{\mu, \mu^{\prime}}$ where $\bar{X}_{\nu}$ and $\bar{Y}_{\mu}$ are eigen-vectors of transposed matrices $M_{1}^{T}$ and $M_{2}^{T}$. Coefficients are defined by the integrals

$$
\begin{gathered}
C_{\mu, \sigma}^{\nu}=2 \int d J \bar{X}_{\nu} Y_{\mu} \frac{\partial}{\partial J}\left[\left(\omega_{H}-\bar{\omega}-\sigma^{*}\right) X_{\sigma}^{*}\right]-\int d J \bar{X}_{\nu} X_{\sigma}^{*} \frac{\partial}{\partial J}\left[\left(\omega_{H}-\bar{\omega}-\mu\right) Y_{\mu}\right]+ \\
\int d J \bar{X}_{\nu} X_{\sigma}^{*} \frac{\left(\omega_{H}-\bar{\omega}-\sigma^{*}\right)}{\rho_{H}^{\prime}} \frac{\partial\left[Y_{\mu} \rho_{H}^{\prime}(J)\right]}{\partial J}-2 \int d J \bar{X}_{\nu} Y_{\mu} \frac{\left(\omega_{H}-\bar{\omega}-\mu\right)}{\rho_{H}^{\prime}} \frac{\partial\left[X_{\sigma}^{*} \rho_{H}^{\prime}\right]}{\partial J}
\end{gathered}
$$




$$
\begin{aligned}
& d_{\nu, \sigma}=2 \pi \lambda \int d J \bar{X}_{n} u X_{\sigma} \int d J^{\prime} \frac{\partial R_{00}\left(J, J^{\prime}\right)}{\partial J} \rho_{o}\left(J^{\prime}\right)-\int d J \frac{\left(\omega_{H}-\bar{\omega}-\sigma\right)}{\rho_{H}^{\prime}} \frac{\partial \rho_{0}}{\partial J} \bar{X}_{\nu} X_{\sigma}, \\
& g_{\sigma, \nu}^{\mu}=i \int d J \bar{Y}_{\mu} X_{\sigma} \frac{\partial}{\partial J}\left[\left(\omega_{H}-\bar{\omega}-\nu\right) X_{\nu}\right]-i \int d J \bar{Y}_{\mu} X_{\sigma} \frac{\left(\omega_{H}-\bar{\omega}-\sigma\right)}{\rho_{H}^{\prime}} \frac{\partial}{\partial J}\left[X_{\nu} \rho_{H}^{\prime}\right], \\
& f_{\mu, \sigma}=4 \pi i \lambda \int d J \bar{Y}_{m} u Y_{\sigma} \int d J^{\prime} \frac{\partial R_{00}\left(J, J^{\prime}\right)}{\partial J} \rho_{o}\left(J^{\prime}\right)-2 i \int d J \frac{\left(\omega_{H}-\bar{\omega}-\sigma\right)}{\rho_{H}^{\prime}} \frac{\partial \rho_{0}}{\partial J} \bar{Y}_{\mu} Y_{\sigma} .
\end{aligned}
$$

Dipole and quadrupole modes interact with each other directly (terms proportional $\left.C_{\mu, \sigma}^{\nu}, g_{\sigma, \lambda}^{\mu}\right)$ and through perturbation of the ground state $\rho_{0}(J)$ (terms proportional to $d_{\nu, \sigma}$, and $\left.f_{\mu, \sigma}\right)$.

Substitute expansion Eqs. (27), (28) in Fokker-Plank equation for $\rho_{0}$

$$
\frac{\partial \rho_{0}}{\partial s}+\gamma_{0} \rho_{0}(J, s)=i \sum_{m}\left(\nu^{*}-\nu^{\prime}\right) b_{\nu}^{*} b_{\nu^{\prime}} \frac{\partial}{\partial J}\left[\rho_{H}^{\prime} X_{\nu}^{*} X_{\nu^{\prime}}\right]+2 i \sum_{m}\left(\mu^{*}-\mu^{\prime}\right) a_{\mu}^{*} a_{\mu^{\prime}} \frac{\partial}{\partial J}\left[\rho_{H}^{\prime} Y_{\mu}^{*} Y_{\mu^{\prime}}\right] .
$$

This allows us to write another set of equations for $d_{\nu, \sigma}$, and $f_{\mu, \sigma}$ :

$$
\begin{gathered}
\dot{d}_{\nu \sigma}+\gamma_{0} d_{\nu \sigma}=-i\left(\nu^{\prime *}-\sigma^{\prime}\right) P_{\nu^{\prime}, \sigma^{\prime}}^{\nu, \sigma} b_{\nu^{\prime}}^{*} b_{\sigma^{\prime}}-2 i\left(\mu^{\prime *}-\lambda^{\prime}\right) Q_{\mu^{\prime}, \lambda^{\prime}}^{\nu, \sigma} a_{\mu^{\prime}}^{*} a_{\lambda^{\prime}}, \\
\dot{f}_{\mu \lambda}+\gamma_{0} f_{\mu \lambda}=\left(\nu^{\prime *}-\sigma^{\prime}\right) F_{\nu^{\prime}, \sigma^{\prime}}^{\mu, \lambda} b_{\mu^{\prime}}^{*} b_{\sigma^{\prime}}+2\left(\mu^{\prime *}-\lambda^{\prime}\right) G_{\mu^{\prime}, \lambda^{\prime}}^{\mu, \lambda} a_{\mu^{\prime}}^{*} a_{\lambda^{\prime}} .
\end{gathered}
$$

Coefficients $P, Q, F$, and $G$ are given by convolution of eigen-vectors and $\rho_{H}^{\prime}$. For example,

$$
P_{\nu^{\prime} \sigma^{\prime}}^{\nu, \sigma}=-\int d J \frac{\partial}{\partial J}\left[\frac{\omega_{H}-\bar{\omega}-\sigma}{\rho_{H}^{\prime}} \bar{X}_{\nu} X_{\sigma}\right] \frac{\partial}{\partial J}\left[\rho_{H}^{\prime} X_{\nu^{\prime}}^{*} X_{\sigma^{\prime}}\right] .
$$

Coefficients $Q, F$, and $G$ have similar structure where some vectors $X$ are replaced by $Y$.

\section{SINGLE MODE}

Let us consider single unstable radial dipole mode $\nu " \equiv \operatorname{Im}[\nu]>0$ taking into account coupling to $\rho_{0}$. The system of equations in this case is 


$$
\dot{b}+\left(i \nu+\gamma_{d}\right) b+i d b=0, \quad \dot{d}+\gamma_{0} d=-2 \nu " P|b|^{2} .
$$

Here $P=P_{\nu, \nu}^{\nu, \nu}$. In the linear approximation the mode is unstable if $\nu ">0$.

In the nonlinear regime, $d$, the momentum of $\rho_{0}$, modifies the linear coherent frequency $\nu$ and can stop and even reverse the sign of the growth rate.

In terms of variables $y=4 \nu " \operatorname{Im}[P]|b|^{2} / \gamma_{0}^{2}, x=\left(2 / \gamma_{0}\right) \operatorname{Im}[d]$, and $s=\gamma_{0} t$, equations

$$
y^{\prime}+\zeta y-x y ; \quad x^{\prime}+x+y=0
$$

depend only on single parameter $\zeta=2\left(\gamma_{d}-\nu "\right) / \gamma_{0}$. Additional to the FP $x=y=0$, there is another FP $x=\zeta, y=-\zeta$. The first FP is stable provided $\zeta>0$ and unstable otherwise. The second $\mathrm{FP}$ is stable for $\zeta<0$ and unstable for $\zeta>0$. Because $|b|^{2}>0$, the second FP exists if $\left(\nu "-\gamma_{d}\right) \nu " \operatorname{Im}[P]>0$. For $\nu ">\gamma_{d}$ this requires $\operatorname{Im}[P]>0$. In the case of linearly stable system, $\gamma_{d}>\nu$ ", the nontrivial FP exists if $\operatorname{Im}[P]<0$ assuming non-zero initial conditions or large fluctuations.

The non-trivial FP corresponds to a limiting cycle where $b$ changes periodically, $b=B e^{i \Omega s}$ with real $\Omega$, while $\dot{d}=0$, and distribution function has a constant distortion $d=i \gamma_{d}-\nu$. For $\Omega$ and $|b|$ we get

$$
\Omega=-\operatorname{Re}(\nu)+\left(\operatorname{Im}[\nu]-\gamma_{d}\right) \frac{\operatorname{Re}[P]}{\operatorname{Im}[P]}, \quad|b|^{2}=\frac{\gamma_{0}}{2 \operatorname{Im}[P]}\left(1-\frac{\gamma_{d}}{\operatorname{Im}[\nu]}\right)
$$

This is the main result of quasi-linear theory: $\rho_{0}$ is distorted by the unstable mode in such a way that the mode is stabilized. A constant distortion changes both rms bunch length and the energy spread.

\section{TWO INTERACTING MODES}

Analysis in more general cases is complicated. Let us consider two dipole modes, one unstable with eigen-values $\nu, \operatorname{Im}[n u]=\nu ">\gamma_{d}$, and another stable mode with eigen-value $\mu$. Effective radiation damping for a mode can be calculated convoluteeing eigen-vectors with the right-hand-side of Eq. (11). The system of equation in this case is

$$
\begin{aligned}
& \dot{b}_{\nu}+\left(i \nu+\gamma_{d}+i d_{\nu, \nu}\right) b_{\nu}+i d_{\nu, \mu} b_{\mu}=0 \\
& \dot{b}_{\mu}+\left(i \mu+\gamma_{d}+i d_{\mu, \mu}\right) b_{\mu}+i d_{\mu, \nu} b_{\nu}=0
\end{aligned}
$$

and four equations for $d$, for example

$$
\dot{d}_{\nu \mu}+\gamma_{0} d_{\nu \mu}=-i \sum_{\nu^{\prime}, \mu^{\prime}}\left(\nu^{\prime *}-\mu^{\prime}\right) P_{\nu^{\prime}, \mu^{\prime}}^{\nu, \mu} b_{\nu^{\prime}}^{*} b_{\mu^{\prime}}
$$



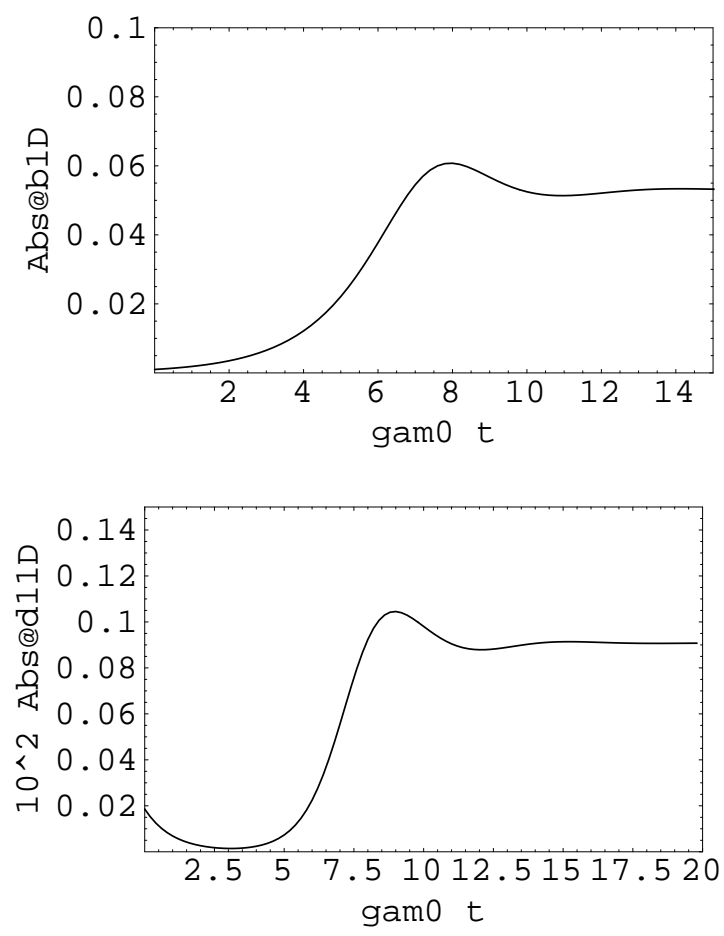

FIGURE 2. Nonlinear saturation of a single dipole mode. Parameters $\lambda=6.8, \alpha=0.4, \mu=1.5$. Eqs.(45), (43) give $|b 1|=0.053,|d|=0.0910^{-2}$.

Let us neglect for a while the non-diagonal terms in Eqs. (46), (47). The mode stability then depends on the dynamic increment $\Gamma_{\nu}=\gamma_{d}-\operatorname{Im}\left[\nu+d_{\nu, \nu}\right]$. Let us take initial conditions where amplitudes $b[0],[0]$ are small. Then $d$ in $\Gamma_{\nu}$ is initially negligible and mode is unstable. The unstable mode leads, first of all, to growth of the diagonal $d_{\nu, \nu}$ described by $\dot{d}_{\nu \nu}+\gamma_{0} d_{\nu \nu}=-2 \nu " P_{\nu, \nu}^{\nu, \nu}\left|b_{\nu}\right|^{2}$. If $\operatorname{Im}\left[P_{\nu, \nu}^{\nu, \nu}\right]>0, d_{\nu \nu}$ is negative and can stabilize mode and reverse sign of $\Gamma_{\nu}$, see Fig. 3.

Due to the same mechanism, $\Gamma_{\mu}$ of stable mode is modified due to variation in time of $\dot{d}_{\mu \mu}+\gamma_{0} d_{\mu \mu}=-2 \nu " P_{\nu, \nu}^{\mu, \mu}\left|b_{\nu}\right|^{2}$. If $\operatorname{Im}\left[P_{\nu, \nu}^{\mu, \mu}\right]<0$, the linearly stable mode can become unstable when linearly unstable mode saturates. After that, their roles interchange and the process can repeat itself. For small $b_{\nu}$ the dominant term defining growth of the mode $\mu$ is $d_{\mu \mu}$ given by $\dot{d}_{\mu \mu}+\gamma_{0} d_{\mu \mu}=-2 \operatorname{Im}[\mu] P_{\mu, \mu}^{\mu, \mu}\left|b_{\mu}\right|^{2}$. If $\operatorname{Im}\left[P_{\mu, \mu}^{\mu, \mu}\right]<0$, the fastest growing mode is the mode with the minimum $\operatorname{Im}[\mu]$, that is, the most stable mode in the linear approximation.

If we take from the beginning $\mu=[\nu]^{*}$, then Eq. (48) is simplified to

$$
\dot{d}_{\nu \nu}+\gamma_{0} d_{\nu \nu}=-2 \nu "\left[P_{\nu, \nu}^{\nu, \nu}\left|b_{\nu}\right|^{2}-P_{\mu, \mu}^{\nu, \nu}\left|b_{\mu}\right|^{2}\right] .
$$

It depends only on $|b|^{2}$ terms. The coefficients $P$ have symmetry

$$
P_{\nu, \nu}^{\nu, \nu}=P_{\mu, \mu}^{\nu, \nu}=\left[P_{\nu, \nu}^{\mu, \mu}\right]^{*}=\left[P_{\mu, \mu}^{\mu, \mu}\right]^{*},
$$




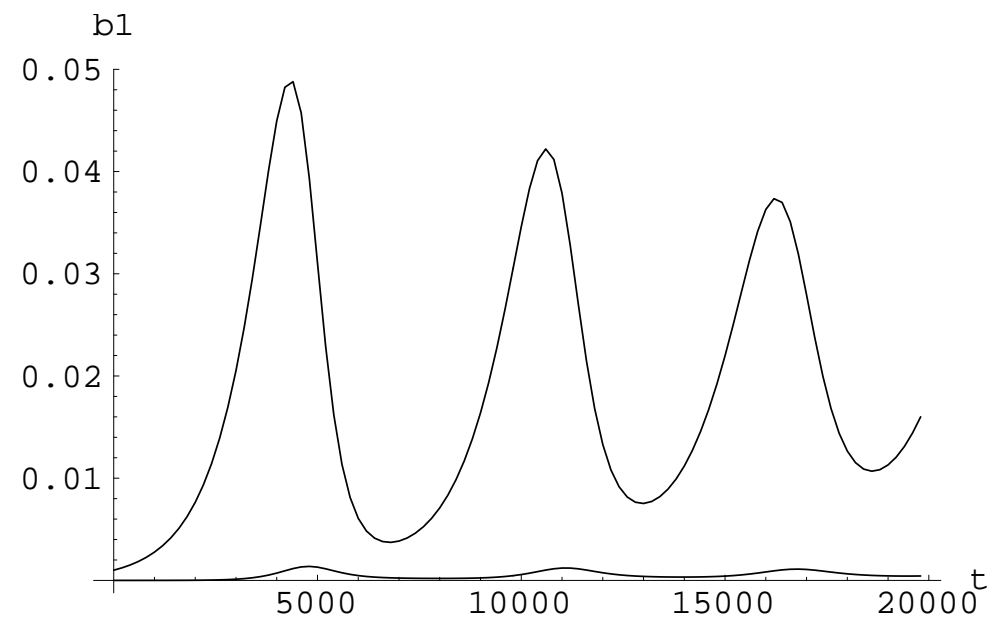

FIGURE 3. Amplitude of linearly unstable dipole mode (upper curve) and dynamic decrement. The growth changes sign when the dynamic increment is larger than linear growth rate, in this case, 0.002 .

$$
P_{\nu, \nu}^{\nu, \mu}=P_{\mu, \mu}^{\nu, \mu}=\left[P_{\nu, \nu}^{\mu, \nu}\right]^{*}=\left[P_{\mu, \mu}^{\mu, \nu}\right]^{*} .
$$

Hence, $\operatorname{Im}\left[P_{\mu, \mu}^{\mu, \mu}\right]<0$ if $\operatorname{Im}\left[P_{\nu, \nu}^{\nu, \nu}\right]>0$. The last requirements is satisfied because $P_{\mu, \mu}^{\mu, \mu}$ is given by the second derivative of a function at the maximum, see Eq. (42).

This preliminary arguments show already that magnitude of the decrement $\gamma_{d}$ is related to the flip-flop dynamics. Let us study in a more systematic way fixed points (FPs) of the problem, considering interaction of the most stable and the most unstable modes. Allowing the limiting cycles, wee assume that $b_{\nu} \propto b_{\mu} \propto e^{-i \Omega s}$ were $\Omega$ is to be defined. Eqs. (46) and (47) give

$$
b_{\mu}=-\frac{d_{\mu \nu} b_{\nu}}{\nu^{*}+d_{\mu \mu}-\Omega-i \gamma_{d}}, \quad\left(\nu-\Omega+d_{\nu \nu}-i \gamma_{d}\right)\left(\nu^{*}-\Omega+d_{\mu \mu}-i \gamma_{d}\right)=d_{\nu \mu} d_{\mu \nu} .
$$

Eq. (49) defines $d$,

$$
d_{\nu \mu}=-\frac{2}{\gamma_{d}}\left[P_{\nu \nu}^{\nu \mu}\left|b_{\nu}\right|^{2}-P_{\mu \mu}^{\nu \mu}\left|b_{\mu}\right|^{2}\right]
$$

Symmetry Eq. (50),(51) gives

$$
\begin{aligned}
& d_{\nu \nu}=d_{\mu \mu}^{*}=-\frac{2}{\gamma_{d}} P_{\nu \nu}^{\nu \nu}\left[\left|b_{\nu}\right|^{2}-\left|b_{\mu}\right|^{2}\right] . \\
& d_{\nu \mu}=d_{\mu \nu}^{*}=-\frac{2}{\gamma_{d}} P_{\nu \nu}^{\nu \mu}\left[\left|b_{\nu}\right|^{2}-\left|b_{\mu}\right|^{2}\right] .
\end{aligned}
$$


Hence, $d_{\nu \mu}=\zeta d_{\nu \nu}$ where $\zeta=P_{\nu \nu}^{\nu \mu} / P_{\nu \nu}^{\nu \nu}$.

The first of Eq. (52) gives for the amplitudes

$$
\left|b_{\mu}\right|^{2}=\left|b_{\nu}\right|^{2} \frac{\left|d_{\nu \mu}\right|^{2}}{\left(\operatorname{Re}\left[\nu+d_{\nu \nu}\right]-\Omega\right)^{2}+\left(\operatorname{Im}\left[\nu+d_{\nu \nu}\right]+\gamma_{d}\right)^{2}} .
$$

The second of Eq. (52) is quadratic equation for $\Omega$,

$$
\Omega=\left(\operatorname{Re}\left[\nu+d_{\nu \nu}\right]-i \gamma_{d}\right) \pm \sqrt{\left|d_{\nu \mu}\right|^{2}-\left(\operatorname{Im}\left[\nu+d_{\nu \nu}\right]\right)^{2}} .
$$

If $\left|d_{\nu \mu}\right|^{2}>\left(\operatorname{Im}\left[\nu+d_{\nu \nu}\right]\right)^{2}$, then $\operatorname{Im}[\Omega]=-\gamma_{d}$ and the trivial FP $b_{\nu}=b_{\mu}=0$ is stable. In the opposite case, $\left|d_{\nu \mu}\right|^{2}<\left(\operatorname{Im}\left[\nu+d_{\nu \nu}\right]\right)^{2}$, FP exists if

$$
\left(\operatorname{Im}\left[\nu+d_{\nu \nu}\right]\right)^{2}=\gamma_{d}^{2}+\left|\zeta d_{\nu \nu}\right|^{2}, \quad \Omega=\operatorname{Re}\left[\nu+d_{\nu \mu}\right] .
$$

In the last case,

$$
\begin{gathered}
\left|b_{\mu}\right|^{2}=\left|b_{\nu}\right|^{2} \frac{\left|\zeta d_{\nu \nu}\right|^{2}}{\left(\operatorname{Im}\left[\nu+d_{\nu \nu}\right]+\gamma_{d}\right)^{2}} \\
d_{\nu \nu}=-\frac{2}{\gamma_{d}} P_{\nu \nu}^{\nu \nu}\left|b_{\nu}\right|^{2}\left[1-\frac{\left|\zeta d_{\nu \nu}\right|^{2}}{\left(\operatorname{Im}\left[\nu+d_{\nu \nu}\right]+\gamma_{d}\right)^{2}}\right]
\end{gathered}
$$

Equation $d_{\nu \mu}=\zeta d_{\nu \nu}$ relates $\operatorname{Red}_{\nu \nu}=\eta \operatorname{Im} d_{\nu \nu}$, where $\eta=\operatorname{Re}\left[P_{\nu \nu}^{\nu \nu}\right] / \operatorname{Im}\left[P_{\nu \nu}^{\nu \nu}\right]$. Then, finally,

$$
\operatorname{Imd}_{\nu \nu}=\frac{1}{|\zeta|^{2}\left(1+\eta^{2}\right)-1}\left[\nu " \pm \sqrt{\gamma_{d}^{2}+|\zeta|^{2}\left(1+\eta^{2}\right)\left[(\nu ")^{2}-\gamma_{d}^{2}\right]} .\right.
$$

Eq. (59) defines $b_{\nu}^{2}$. It has to be positive what impose some constrain on parameters $P$ and $\gamma_{d}$.

Computer simulations based on Eqs. (46-48) confirmed these results. Fig. 4 depicts saw-tooth behavior in the system of two dipole modes interacting through perturbation of distribution function $\rho_{0}$. The phases of oscillations of two modes are shifted and the moment $d_{\nu \nu}$ of $\rho_{0}$ also oscillates in time. The oscillations are anharmonic and anharmonicity depends on the initial conditions and radiation damping.

In the same way interaction of other modes can be explored. Fig.5 depicts interaction of two linearly unstable modes, one quadrupole and another dipole. The nonlinear interaction leads to saturation of both modes.

Fig.6 shows interaction of three modes: linearly unstable and stable dipole modes and linearly unstable quadrupole mode. When current increases, the quadrupole mode become linearly unstable. At the same time, increasing nonlinearity of the potential well may suppress relaxation oscillations of the dipole modes. Interaction of these mode with dipole modes may lead to their stabilization while saw-tooth behavior may be preserved in quadrupole radial modes.

Certainly, more simulations should be carried out in a consistent way. This would allow direct comparison with the experimental data. 

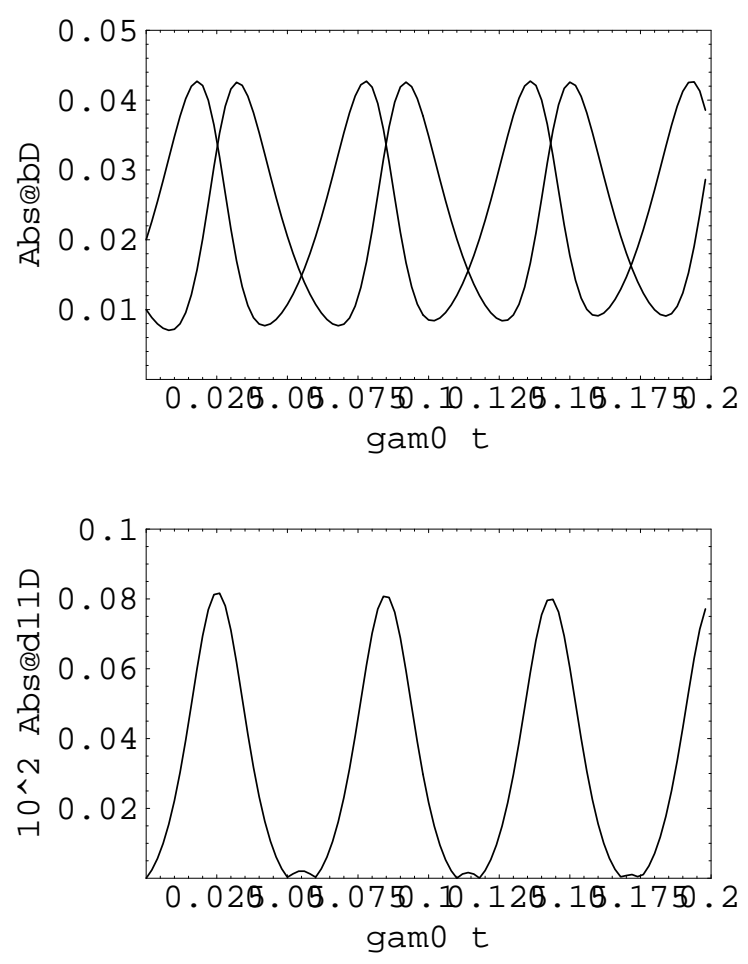

FIGURE 4. Coupled oscillations of two dipole modes. One is linearly unstable and another one is the most linearly stable. Parameters $\lambda=6.88, \alpha=0.4, \mu=1.5, \gamma_{0}=10^{-5}$. Initial conditions are $b_{1}=210^{-2}, b_{2}=10^{-2}$, all $d=0$.

\section{CONCLUSION}

Dynamics of the system in the nonlinear regime above the threshold of instability may be quite complicated and substantially depends on the impedance, radiation damping, and beam current. Additional to already known mechanisms of linear mode coupling and Baartman-Dyachkov mechanism, both of which require high currents leading to large coherent tune shifts or appearance of the second minima in the self-consistent potential, there is another mechanism of nonlinear mode coupling. We explore the last mechanism and demonstrated that it may lead to the saw-tooth oscillations for beam current close and above the threshold of microwave instability. Numerical results are qualitatively similar to that obtained in reference [7] but, from our point of view, allows more systematic approach to the nonlinear collective phenomena. Application of this approach to other cases mentioned in the introduction will be reported elsewhere.

\section{REFERENCES}

1. Krejcik, P. et al., Proc. IEEE Part. Accel. Conf., Washington D.C., 1993, p. 3240. 
B.V. Podobedov and R.H. Siemann, Signals from Microwave Unstable Beams in the SLC Damping Rings, PAC99, 1999, New York.

2. Haissinski, J. Nuovo Cimento, 1973, 18B, pp. 72.

3. Baartman, R., and Dyachkov, M. Proc. IEEE Part. Accel. Conf., Dallas, 1995.

4. Oide, K., "A New Mechanism of Longitudinal Single Bunch Instability in Storage Ring", KEK, 1995 (unpublished).

5. O’Neil, T. Phys. Fluids 1965, 8, pp. 2255.

Chin Y., and Yokoya, K., Nucl Instr. and Methods, 1984, 226, 223-249.

Morales,G.T. and O'Neil,T. Phys. Rev. 1965, 28, pp. 417.

Schonfeld, J., Ann. Phys., 1985, 160, pp. 149.

Meller, R.E., Ph. D. Thesis, "Statistical Method for Nonequilibrium Systems with Application to Accelerator Beam Dynamics", Cornell, 1986.

Heifets, S.A., Phys. Rev. 1996, 54, p.2889.

6. J. Byrd, F. Zimmermann Private communication

7. Stupakov, G.V.,. Breizman, B.N, and Pekker, M.S., SLAC-PUB-7377, 1996 


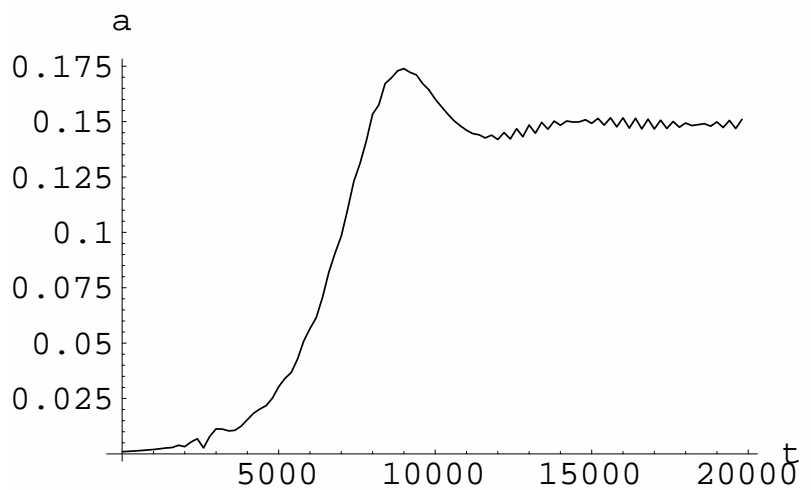

$\mathrm{b}$

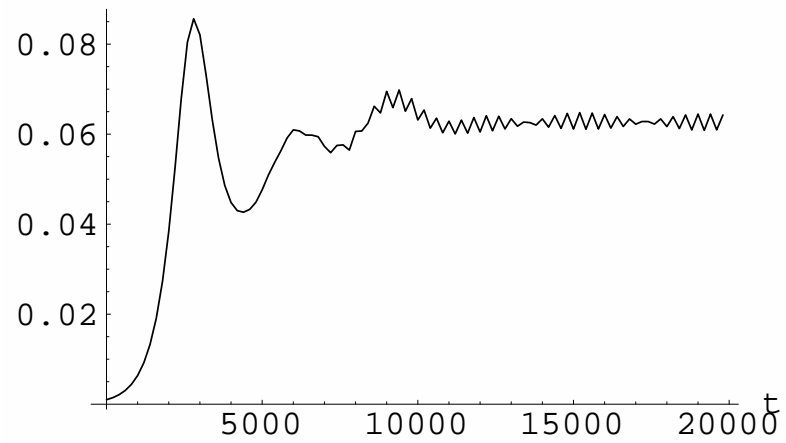

Out [58]= Graphics

lambda $=6.88447$ alpha=0.9 mu=1.5

gam $0=0.001$ gamdip $=0.0001$ gamquad $=0.0001$

Dipole + Quadrupole modes, both

linearly unstable. Stabilization is due to nonlinear interaction.

FIGURE 5. 

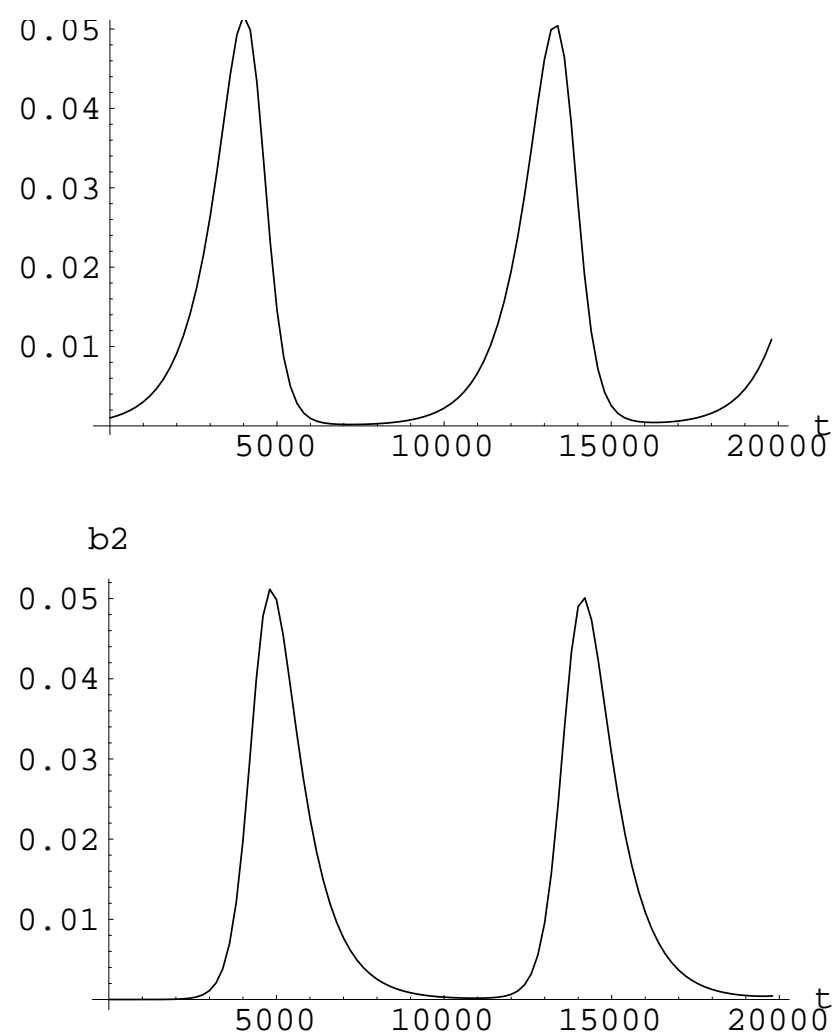

a

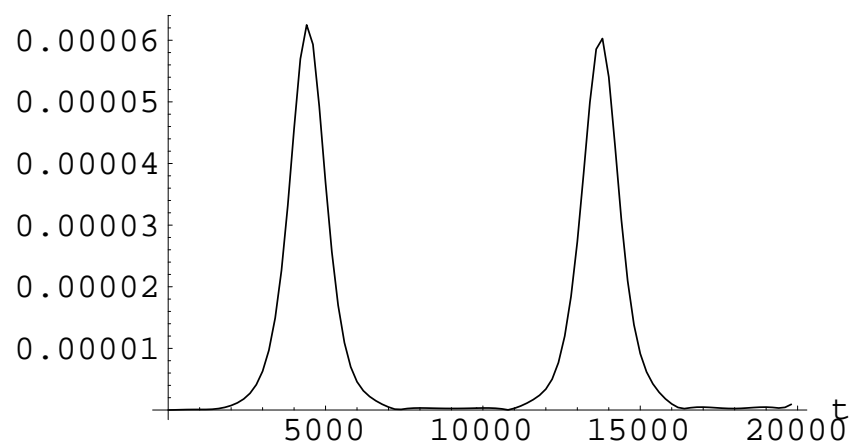

b1

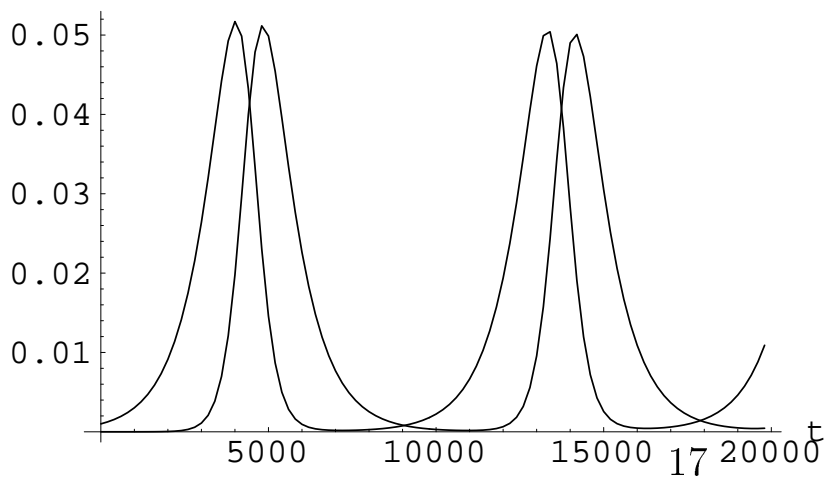

FIGURE 6. Interaction of two dipole modes and one quadrupole mode. One of the dipole modes is linearly unstable, another is the most linearly stable mode. Quadrupole mode is linearly stable. 